\title{
Dynamic simulation of a combined cycle for power plant flexibility enhancement
}

\author{
Adrien Reveillere ${ }^{1, *}$, Martin Longeon ${ }^{1}$ and Iacopo Rossi ${ }^{2}$ \\ ${ }^{1}$ Siemens PLM 69006, Lyon (France) \\ ${ }^{2}$ University of Genoa, 16145, Genoa (Italy)
}

\begin{abstract}
System simulation is used in many fields to help design, control or troubleshoot various industrial systems. Within the PUMP-HEAT H2020 project, it is applied to a combined cycles power plant, with innovative layouts that include heat pumps and thermal storage to un-tap combined cycle potential flexibility through low-CAPEX balance of plant innovations. Simcenter Amesim software is used to create dynamic models of all subsystems and their interactions and validate them from real life data for various purpose. Simple models of the Gas Turbine (GT), the Steam loop, the Heat Recovery Steam Generator (HRSG), the Heat Pump and the Thermal Energy storage with Phase Change material are created for PreDesign and concept validation and then scaled to more precise design. Control software and hardware is validated by interfacing them with detailed models of the virtual plant by Model in the Loop (MiL), Software in the Loop (SiL) and Hardware in the Loop (HiL) technologies. Unforeseen steady state and transient behaviours of the powerplant can be virtually captured, analysed, understood and solved. The purpose of this paper is to introduce the associated methodologies applied in the PUMP-HEAT H2020 project and their respective results.
\end{abstract}

\section{Introduction}

A Combined Cycle Power Plant (CC) [1] converts energy contained within the fuel into electrical energy, through two different cycles in one simple plant, to improve efficiency. First fuel is burnt to feed a Gas Turbine (GT) in a Brayton cycle, then heat is recovered from waste exhaust to heat up steam to feed steam turbines in a Rankine cycle. In the particular case of cogenerative $\mathrm{CC}$, a part of the energy recovered from the exhaust can be used to supply heat to District Network in the Cogeneration mode [2].

Because of decarbonisation necessity [3], CC plant number is going to decrease in the incoming future while renewable energy will increase. However, $\mathrm{CC}$ technology should keep an interest to compensate the production fluctuation of renewable energy [4]. This can only be true if CC plants turn out to be more flexible and more adaptive to the market fluctuation.

\footnotetext{
*Corresponding author: adrien.reveillere@siemens.com
} 
Solutions exist [5] such as integrating Heat Pump [6] and energy storage [7] in CC plants, so that the power plant is able to pick up excess energy during high renewable energy production and use it later when it is required.

The presented work has been carried out within the H2020 EU Project Performance Untapped Modulation for Power and Heat via Energy Accumulation Technologies (PUMP-HEAT). In the presented context, the PUMP-HEAT project [8] studies two main layouts of Heat Pump and Energy storage in CC. The first layout considered, a Power Oriented one, conditions the Gas Turbine inlet temperature [9] to adapt the electricity production to the market demand. The second layout studies the integration of Heat Pump and Latent Heat storage in Cogenerative $\mathrm{CC}$ to extract heat from condensate to provide additional heat to District Network. Both solutions will be prototyped, the second one will be tested at TRL 6 in the demosite located in Turin (Italy) on the Moncalieri 400 MW CC. The plant is equipped with a Siemens V94.3A with annular combustion chamber.

System dynamic models developed with Simcenter Amesim are involved at different stages in this project. First during the predesign stage the concept has been modelled to confirm its interest and to study its dynamic behaviour. Secondly, as introduced by the authors [10], this prototype will include a predictive controller where current scheduling, constraints and plant status will interact to achieve the best thermo-economic efficiency. The controller will be developed in Matlab/Simulink and will integrate Simcenter Amesim linearized model. Finally, model of the plant developed in Simcenter Amesim will be interfaced with the Matlab/Simulink MPC to validate it

\section{Design and Concept}

Early in the Pump Heat project, a need to assess system simulation's capacity to reproduce the power plant behaviour was identified. The model in Figure 1 shows

- The gas turbine system (top left)

- The heat recovery Steam Generator (column of heat exchangers) on the left

- The complete steam loop with the three pressure levels (high pressure in red, intermediate pressure in green, low pressure in blue)

- The three steam turbines on the right

This model has been fully validated in steady state using measurements from a known operating point of the power plant.

To enhance the flexibility of the power plant, one specific designed layout focuses on retrieving heat from the steam loop and transferring either to the district heat network or to the thermal storage unit. Here as well, Simcenter Amesim is used in predesign phase to assess the performance of the system. The model in Figure 2 shows:

- The district heating network receiving heat (blue)

- A water intermediate loop (green)

- The butane heat pump (red)

- The steam line where heat is taken (brown)

This model has been validated from the known design from the manufacturer: thermodynamic cycle, exchanged powers and pressure and temperature levels. 


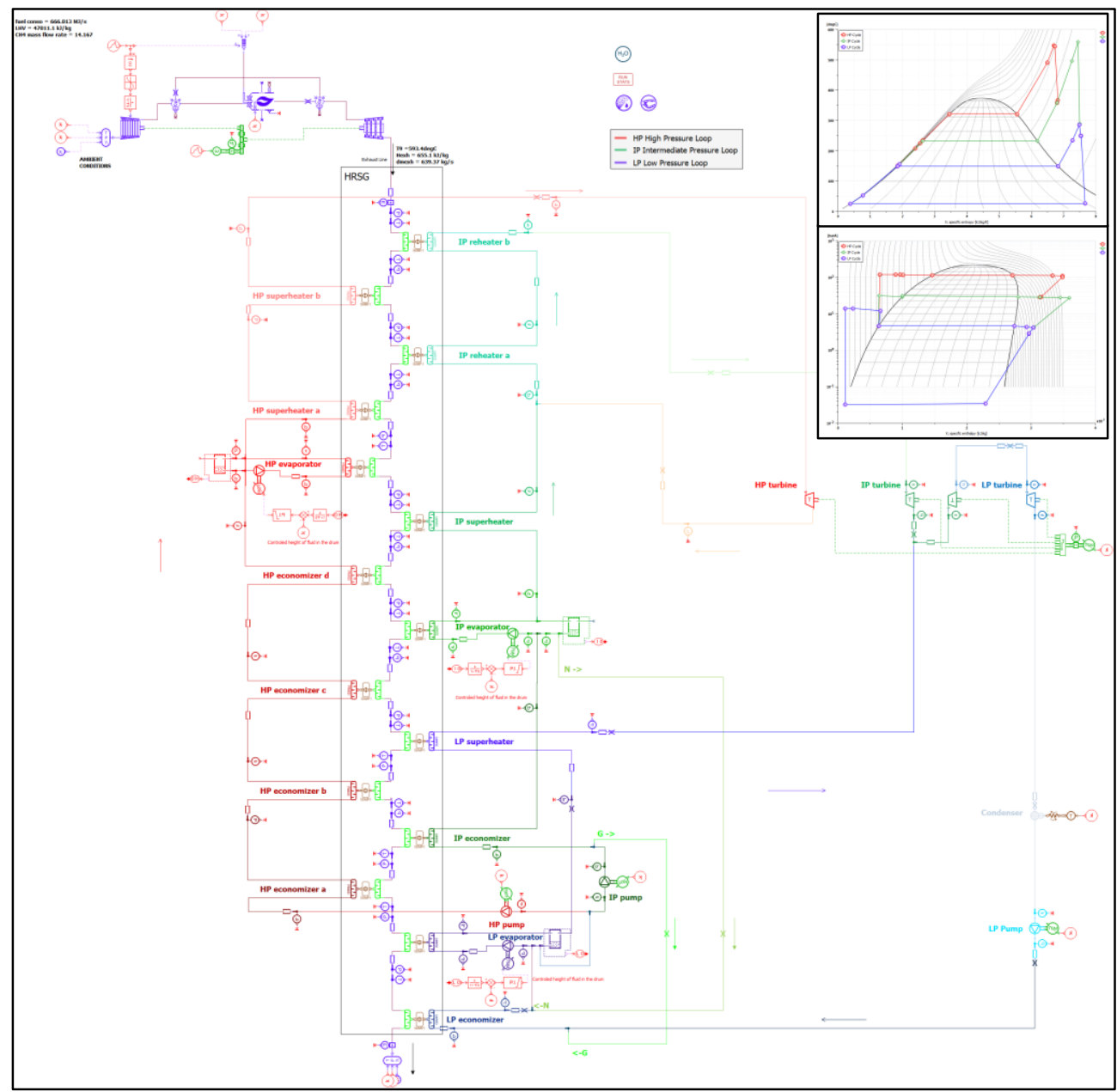

Figure 1: Global combined cycle power plant model

\section{Control Software}

The developed control software has been already presented in the previous paper [10] in its main principles: a framework based on a hierarchic approach was presented. A Model Predictive Controller (MPC) governs the power plant with real time resolution for plant operations. The MPC encloses a linear model of heat pump and storage for the correct management in real time.

A virtual heat pump and storage system simulation dynamic model is created using Simcenter Amesim. It is continuously calibrated with real life up-to-date data whose completeness and accuracy are improving during the project at the same time as the MPC:

- Predesign phase: thermodynamic cycle of the heat pump, steady state power, pressure and temperature levels are available

- Design phase: Subsystems datasheets (compressor, heat exchangers, expansion device) are available

- Test phase: steady state and transient test data are available for subsystems (heat exchangers) and complete heat pump system are available 


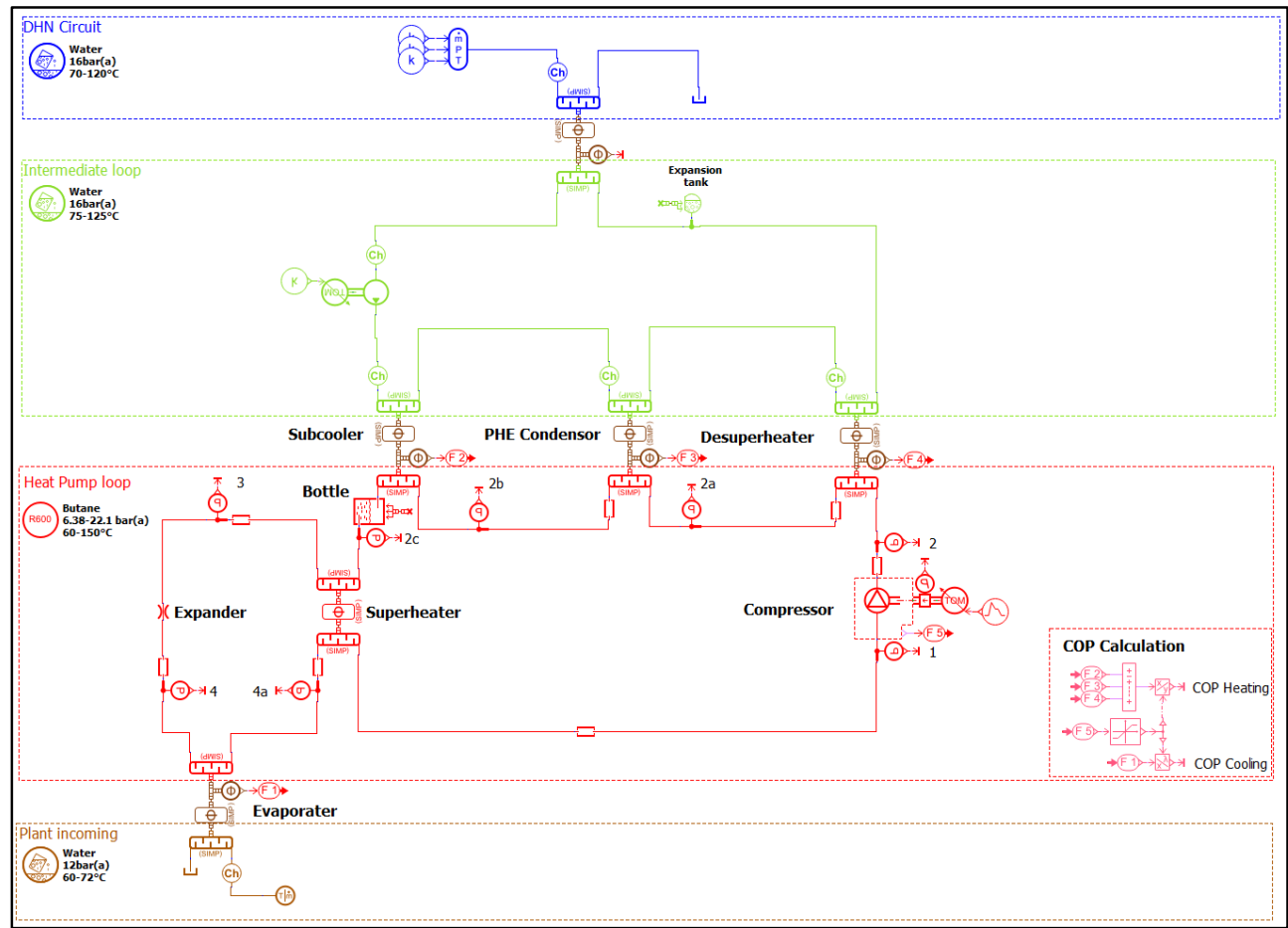

Figure 2: Heat pump model for the cogenerative layout

- Operational phase: measurements (powers, pressure and temperatures) of the operation of the heat pump are available

This model serves different purpose. Once linearized, it is integrated into the MPC to bring the predictivity. It will be used in the validation phase of the control system by interfacing the MPC with Simcenter Amesim which emulates the real system behaviour in real time using MiL, SiL and HiL technologies.

The workflow for the MPC design and validation is as follows:

- The heat pump model is created with Simcenter Amesim. This non-linear model is continuously calibrated with real life data starting from the predesign phase, then design phase test phase and finally operational phase.

- Using a Jacobian matrix evaluation around a standard operating point, the model is linearized. Thus, we create three matrices A B and C such as:

$$
\left\{\begin{array}{l}
\dot{X}=A \cdot X+B . U \\
Y=C . X
\end{array}\right.
$$

Where $X$ is a vector of 32 state variables, $X$ is its time derivative, $U$ is a vector of one input and $Y$ a vector of one output.

- The implemented MPC is based on the principle of receding horizon control, where a current information of the plant is required for prediction and control. By doing so, it is implicitly assumed that the input $u(k)$ cannot affect the output $y(k)$ at the same time - thus $\mathrm{D}=0$ in the plant model [11].

- These matrices are injected into the Matlab/Simulink code of the MPC algorithm to be run every second. 
- Firstly, MiL simulation. The MPC Matlab/Simulink model, included its linear model of the plant, is exported in $\mathrm{C}$ code and imported into the Simcenter Amesim model of the Heat Pump as a control block. In this phase, Simcenter Amesim runs autonomously both the virtual plant and its control systems on any desktop.

- Secondly, SiL simulation. The control system and the virtual plant are run together as a cosimulation between Simcenter Amesim and Matlab/Simulink. Given a communication time step at which they exchange the $U$ and $Y$ information, each of the two software runs its own system, Simcenter Amesim predicts the behavior of the plant and the MPC gives the command.

- Finally, HiL simulation. The same cosimulation is run directly on the industrial control machine the will be implemented on site. This machine has been designed especially for this phase, making sure that it has enough power to run such a cosimulation.

The objective here is to validate the models step by step, as close as possible to the final implementation of the control system. Using this approach, associated with a continuous improvement of the plant model, any deviation with the desired behaviour of the whole system is detected as soon as possible leading to early and less expensive mitigation action if necessary.

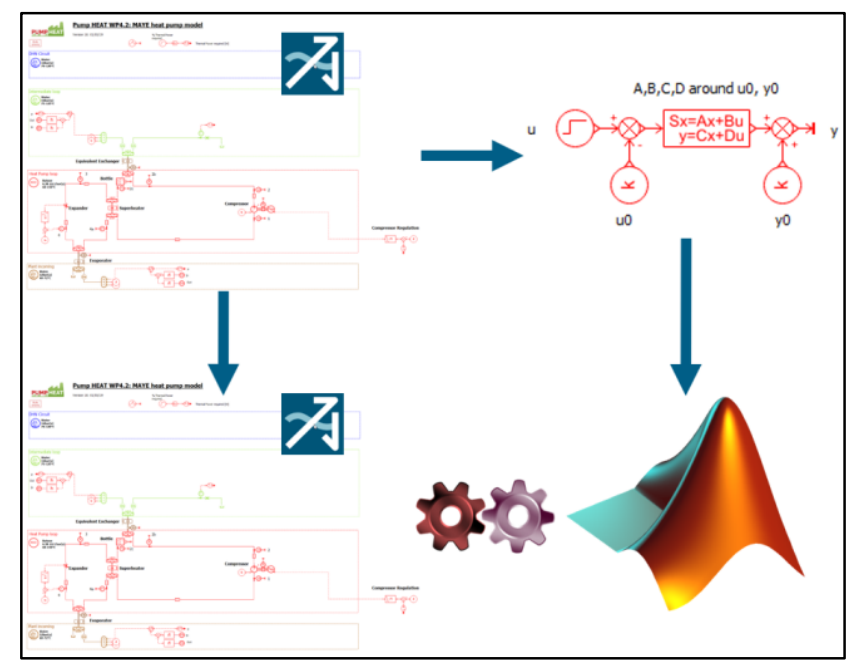

Figure 3: Virtual plant model and its usage in the MPC and for MPC testing

\section{Conclusion}

The paper introduces a model to represent a full scale CHP combined cycle developed within Amesim and the control developed within Matlab/Simulink. The controller is interfaced with the system. The interface is created in Amesim and the system model, which has been linearized and implemented into the Matlab/Simulink controller. The control is based on a MPC linear architecture and the validation process of the control that will be adopted is described. This paper introduces the topic: the MPC controller has been described in [12], whilst specification for its real implementation are given in [13]. 


\section{Nomenclature}

$\begin{array}{llll}\text { CC } & \text { Combined Cycle Power Plant } & \text { MiL } & \text { Model in the Loop } \\ \text { GT } & \text { Gas Turbine } & \text { MPC } & \text { Model Predictive Controller } \\ \text { HiL } & \text { Hardware in the Loop } & \text { SiL } & \text { Software in the Loop } \\ \text { HRSG } & \text { Heat Recovery Steam Generator } & & \end{array}$

\section{Acknowledgments}

PUMP-HEAT has received funding from the European Union's Horizon 2020 research and innovation programme under grant agreement No 764706

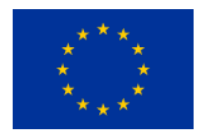

\section{References}

[1] Kelhofer R. et al., "Combined-Cycle Gas \& Steam Turbine Power Plants", McGrawHill (2009).

[2] Meherwan P. et al., "Handbook for Cogeneration and Combined Cycle Power Plants", ASME Press - Technology \& Engineering (2002).

[3] GIEC (IPCC), "Fifth Assessment Report of the Intergovernmental Panel on Climate Change" (2014)

[4] Devlin J. et al., "Gas generation and wind power: A review of unlikely allies in the United Kingdom and Ireland" Renewable and Sustainable Energy Reviews 70, pp. 757-768 (2017)

[5] Guédez R. et al., "Combined cycles integrated with a heat pump and thermal energy storage system for air pre-cooling - a techno-economic analysis", 9th International Gas Turbine Conference, 10-11 October 2018, Brussels, Belgium, Paper ID Number: 57-IGTC18

[6] Bosser S. et al., "High temperature heat pump - a novel approach to increase flexibility and efficiency of CCGT and CHP power plants" 9th International Gas Turbine Conference, 10-11 October 2018, Brussels, Belgium, Paper ID Number: 56IGTC18

[7] Gkoutzamanis V. et al., "Thermal energy storage for gas turbine power augmentation", Global Power and Propulsion (GPPS) Society, Proceedings of Zurich 2019, GPPS-TC-2019-72 (2018)

[8] https://www.pumpheat.eu/

[9] Rossi I. et al., "Ambient temperature impact on micro gas turbines: Experimental characterization", Acta Polytechnica CTU Proceedings, 20:78-85, https://doi.org/10.14311/APP.2018.20.0078 (2018)

[10] Rossi I. et al., "Intelligent predictive control of a pump-heat combined cycle: Introduction and first results", 9th International Gas Turbine Conference, 10-11 October 2018, Brussels, Belgium, Paper ID Number: 59-IGTC18

[11] Wang L. et al., "An improved structure for model predictive control using nonminimal state space realization" Journal of process control, vol.16, pp 355-371

[12] Rossi I. et al., "Development and installation of control system for a test rig interconnecting a micro Gas Turbine, a Heat Pump and a PCM Storage", SUPEHR

[13] Rossi I. et al., "Flexibilization of Gas Turbine Combined Cycle via Heat Pump: development of control logics via Software-in-the-Loop application", SUPEHR 